\title{
Cancer vaccine field gets shot of optimism from positive results
}

An experimental cancer vaccine has for the first time been shown to significantly extend the lives of men with prostate cancer, Seattle-based Dendreon announced in February. The vaccine, Provenge, is based on dendritic cells, which help the immune system recognize tumors.

"If these results are true, this represents the first proof of principle of a dendritic [cell] vaccine altering the natural history of a solid tumor," says Philip Kantoff, chief of solid tumor oncology at the Dana-Farber Cancer Institute in Boston, who was not involved with the study.

Cancer vaccines vary widely: they may employ whole proteins, tumor antigens, dendritic cells, killed tumor cells or tumor lysates. The goal of the vaccines is to prime the patient's immune system to recognize and destroy the tumor without harming normal cells.

Provenge carries the patients' own dendritic cells engineered to express a protein found on about $95 \%$ of prostate cancer cells. It is one of more than 15 vaccines in trials for a range of cancers, including lymphoma, melanoma, breast, lung and colorectal cancers, but the first to increase lifespan in advanced cancer patients.

For years cancer vaccines have been dogged by questions and controversy (Nat. Med. 10, 3; 2004). One ongoing concern is a lack of stan-

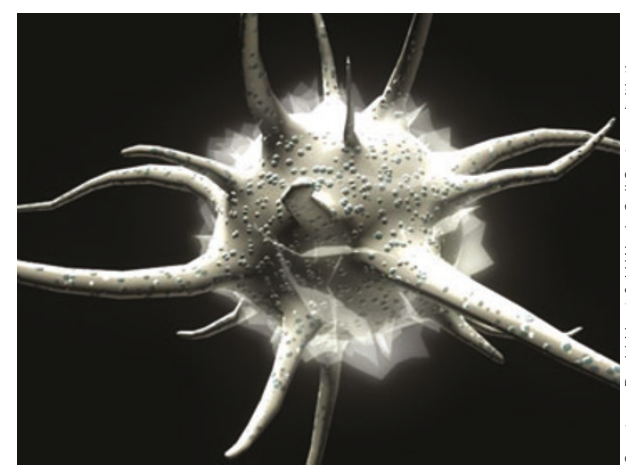

Rays of hope: Scientists have shown that dendritic cell vaccines can help cancer patients live longer.

dardized vaccine production methods, says Nina Bhardwaj, director of the tumor vaccine program at New York University School of Medicine.

There are also questions about which cells and antigens to use in vaccines and the mode and frequency with which they should be administered. For example, mature dendritic cell vaccines seem to produce the best immune response when given either subcutaneously or intradermally, but not intravenously (Blood 10, 2235-2246; 2004). Researchers are also trying to determine whether vaccines will be need to be designed for specific cancers, or whether a universal vaccine could produce a robust immune response.

The Provenge trial has raised several questions of its own: $34 \%$ of men given Provenge, compared with $11 \%$ on placebo, were alive three years on, and lived 4.5 months on average longer. But paradoxically, the vaccine does not delay 'time to progression,' a measure of efficacy used in most cancer trials to denote the time before a tumor begins growing again, and Dendreon's primary endpoint in this trial.

"Because of this, we were all somewhat pleasantly surprised that Provenge showed a survival benefit," says E. Roy Berger, director of the Prostate Cancer Consultation and Treatment Service in East Setauket, New York.

The vaccine only benefited a subgroup of men at earlier stages of the disease in a previous shorter trial, but even men with aggressive disease lived longer in this trial. One reason for that discrepancy may be that immunotherapy does not work as quickly as chemotherapy, which has a direct, cytotoxic effect, says Berger. "Cancer vaccines prime the immune system to attack cancer, a slower, more indirect process," he says. This may mean that survival, and not time to progression, is a better measure of efficacy for cancer vaccines, Berger suggests.

Vicki Brower, New York

\section{Aggressive HIV strain sets off dubious public health measure}

The media frenzy that erupted over the announcement in February of a highly virulent and multidrug-resistant HIV strain

has triggered a somewhat thorny debate on the

future of HIV surveillance and prevention.

Scientists and activists criticized the New York City Department of Health and Mental Hygiene for prematurely inflating the significance of a single case of HIV. The department is once again inviting controversy by promoting a public health measure that some experts say is ineffective.

In a departure from current practice, city health commissioner Thomas Frieden is urging doctors and laboratories to provide data on multidrug resistance in newly diagnosed HIV patients. Officials are also debating whether to enforce a rule requiring labs to provide that information. The data would help "to both guide treatment and help us monitor the AIDS epidemic," Frieden says.

Under New York state law, all laboratories must inform the city and state health departments of new HIV infections, AIDS diagnoses, viral load results and T-cell counts. Requiring reports of multidrug resistance would not need changes in legislation but would entail the creation of a new rule, which is under discussion, says Frieden. Officials are also discussing how best to tackle the technical details of reporting drug resistance. The recently erected citywide Commission on HIV/AIDS is also expected to release a report outlining strategies on HIV/AIDS prevention, treatment and control in a few months.

New York may become the first US state to require AIDS drug resistance data. The Minnesota Department of Health also in February e-mailed 300 infectious disease specialists asking them to consider resistance testing in newly diagnosed patients, but the request is by no means a mandate. Across the US, the so-called 'AIDS superbug' propelled public health officials to check locally for evidence of similar strains, and to reissue public health messages about HIV. But the US Centers for Disease Control and Prevention has no plans to implement federal public health measures until more information emerges about the lone New York case, says Karlie Stanton, a spokeswoman for the agency's HIV prevention office.

Some experts say although drug-resistant HIV strains may be on the rise, testing for resistance is not useful. The tests can help decide treatment regimens, but they are expensive, time consuming and do not always detect resistance in HIV patients who have lived with the virus a long time. "Until we have sufficient data to suggest that the prevalence [of drug-resistant strains] is five percent or higher in a population, it is a guess on the part of clinicians as to the utility of the test," says Tracy Sides, an HIV epidemiologist at the Minnesota Department of Health.

With fewer federal dollars supporting AIDS work, the best use of the resources is to boost existing HIV prevention infrastructure rather than require resistance tests that may not be entirely effective, says Julie Davids, executive director of the Community HIV/ AIDS Mobilization Project, a New Yorkbased advocacy organization. "Clinicians, epidemiologists, researchers and activists should together discuss what measures are most cost effective," she says.

Scientists in January detected the potent new HIV strain that progressed to full-blown AIDS within four months. HIV infections normally take about ten years to turn into AIDS.

Paroma Basu, Madison 